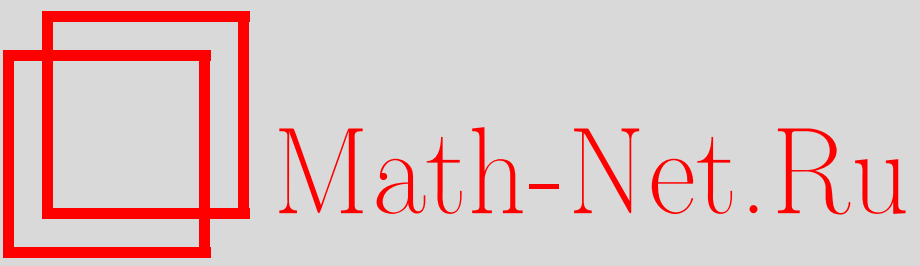

А. Ю. Смыслов, Расчет напряжения осадки круглой шайбы из пористого материала, Вестн. Сам. гос. техн. ун-та. Сер. Физ.-мат. науки, 2002, выпуск 16, 90-92

DOI: https://doi.org/10.14498/vsgtu103

Использование Общероссийского математического портала Math-Net.Ru подразумевает, что вы прочитали и согласны с пользовательским соглашением

http: //www . mathnet.ru/rus/agreement

Параметры загрузки:

IP : 35.173 .219 .12

26 апреля 2023 г., 17:40:07

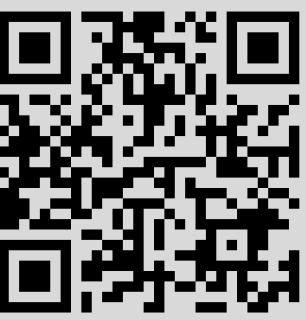




\section{РАСЧЕТ НАПРЯЖЕНИЯ ОСАДКИ КРУГЛОЙ ШАЙБЫ ИЗ ПОРИСТОГО МАТЕРИАЛА}

Рассматривается напряженно-деформированное состояние круглой шайбы из пористого пластического материала при сжатии между двумя матрицами. Получены уравнения, описываюшие изменение пористости и размеров иайбы в процессе сжатия в зависимости от приложенного давления.

Рассматривается напряженно-деформированное состояние круглой шайбы из пористого материала при сжатии между двумя плоскими матрицами. В $[1,2]$ рассматривались закономерности осадки полосы из пористого пластического материала. Основной задачей теоретического исследования обработки давлением пористых материалов является установление зависимостей: давление-пористость, давление-размеры изделия. В данной статье получена полная система уравнений, описывающих изменение пористости и размеров шайбы в зависимости от среднего давления.

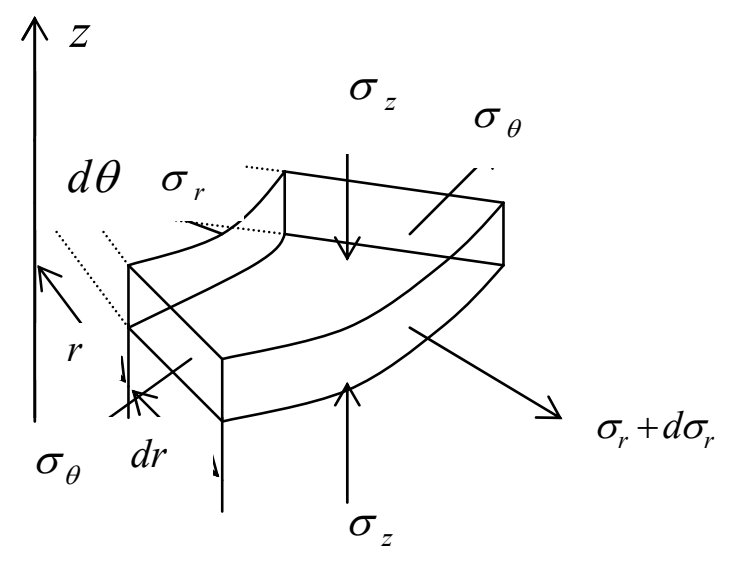

Р и с. 1. Напряжения, действующие на элемент объема шайбы при осадке между двумя матрицами
На рис. 1 изображен элемент объема шайбы, ограниченный двумя радиальными сечениями, образующими угол $d \theta$ и двумя цилиндрическими поверхностями с радиусами $r$ и $r+d r$. Следуя [1], воспользуемся допущениями: $\sigma_{r}$ и $\sigma_{\theta}$, действующие в плоскостях, параллельных боковым граням пластины, не изменяются по ее толщине. Указанные напряжения и напряжение $\sigma_{z}=-p$, действующее на поверхности контакта с матрицами, являются главными напряжениями, входящими в условие пластичности сжимаемого материала. Касательное напряжение $\tau$ равно пределу пла-

стичности деформируемого металла при сдвиге.

Условие равновесия компонентов сил, действующих на элемент объема вдоль оси $x$, приводит к уравнению [1]

$$
h d \sigma_{r}-2 \tau d r=0,
$$

где $h$ - толщина шайбы. Уравнение (1) интегрируется при сделанном предположении относительно касательного напряжения $\tau$.

Далее воспользуемся условием пластичности пористых тел [3], которое для рассматриваемого напряженного состояния записывается в форме

$$
\left(\sigma_{r}-\sigma_{\theta}\right)^{2}+\left(\sigma_{\theta}-\sigma_{z}\right)^{2}+\left(\sigma_{z}-\sigma_{r}\right)^{2}+3 \alpha\left(\sigma_{r}+\sigma_{\theta}+\sigma_{z}\right)^{2}=2 \beta \sigma_{0}^{2},
$$

где $\sigma_{0}$ - предел пластичности межпорового материала, который считается идеальнопластическим, так что $\sigma_{0}=$ const ; $\alpha$ и $\beta-$ функции пористости $c$, полученные в [3] в виде

$$
\alpha=\frac{c}{2(3+2 c)}, \beta=\frac{3(1-c)^{2}}{3+2 c} .
$$

Пористость считается равномерно распределенной по объему деформируемого материала.

Согласно (2) предел пластичности пористого металла при сдвиге $\tau_{0}=\sigma_{0} \sqrt{\beta / 3}$. Подставляя в (1) значение $\tau=\tau_{0}$, получим дифференциальное уравнение, решение которого с граничным условием $\sigma_{r}=0$ при $r=a$ дает

$$
\sigma_{r}=\tau_{0} \lambda \xi, \lambda=\frac{a}{h}, \xi=\frac{r}{a}-1,
$$

где $a$ - радиус шайбы.

Соотношения ассоциированного закона деформирования при условии пластичности (2) записываются в виде [3] 


$$
\begin{aligned}
& d \varepsilon_{r}=d \lambda\left[\sigma_{r}-(1-3 \alpha) \frac{\sigma_{r}+\sigma_{\theta}+\sigma_{z}}{3}\right] ; \\
& d \varepsilon_{z}=d \lambda\left[\sigma_{z}-(1-3 \alpha) \frac{\sigma_{r}+\sigma_{\theta}+\sigma_{z}}{3}\right] ; \\
& d \varepsilon_{\theta}=d \lambda\left[\sigma_{\theta}-(1-3 \alpha) \frac{\sigma_{r}+\sigma_{\theta}+\sigma_{z}}{3}\right],
\end{aligned}
$$

где $d \varepsilon_{r}, d \varepsilon_{\theta}$ и $d \varepsilon_{z}$ - приращения пластических деформаций. Упругие деформации считаются пренебрежимо малыми.

Будем считать, что $d \varepsilon_{\theta} \approx 0$. Тогда из последнего соотношения (5) получаем выражение

$$
\sigma_{\theta}=\frac{1-3 \alpha}{2+3 \alpha}\left(\sigma_{r}+\sigma_{z}\right),
$$

с учетом которого условие пластичности (2) и соотношения (5) соответственно преобразуются к виду

$$
\begin{gathered}
\left(\sigma_{r}-\sigma_{z}\right)^{2}+4 \alpha_{1} \sigma_{r} \sigma_{z}=4\left(1-\alpha_{1}\right) \tau_{0}^{2} \\
d \varepsilon_{r}=d \lambda \frac{\sigma_{r}-\left(1-2 \alpha_{1}\right) \sigma_{z}}{2\left(1-\alpha_{1}\right)}, d \varepsilon_{z}=d \lambda \frac{\sigma_{z}-\left(1-2 \alpha_{1}\right) \sigma_{r}}{2\left(1-\alpha_{1}\right)}
\end{gathered}
$$

где

$$
\alpha_{1}=\frac{9 \alpha}{2(1+6 \alpha)} .
$$

Решение (7) относительно $\sigma_{z}$ с учетом (4) для $\sigma_{r}$ дает

$$
\sigma_{z}=\tau_{0}\left[\left(1-2 \alpha_{1}\right) m \xi-2\left(1-\alpha_{1}\right)\left(1-\alpha_{1} m^{2} \xi^{2}\right)\right] .
$$

Одной из задач теоретического исследования процессов обработки давлением пористых материалов является определение пористости и размеров изделия в зависимости от деформирующих усилий.

Вычислим средние напряжения, действующие на пластину:

$$
\bar{\sigma}_{z}=\frac{1}{a} \int_{0}^{a} \sigma_{z}(r) d r .
$$

Выражение для $\bar{\sigma}_{z}$ будет менее громоздким, если правую часть равенства (10) приближенно заменить линейной зависимостью

$$
\sigma_{z}=\tau_{0}\left\{\left[\left(1-2 \alpha_{1}\right) m-2 \sqrt{1-\alpha_{1}}\left(1-\sqrt{1-\alpha_{1} m^{2}}\right)\right] \xi-\sqrt{1-\alpha_{1}}\right\} .
$$

С учетом (4) и (12) средние напряжения определяются по (11) в виде

$$
\bar{\sigma}_{z}=-\frac{\tau_{0}}{2}\left[\left(1-2 \alpha_{1}\right) m+2 \sqrt{1-\alpha_{1}}\left(1+\sqrt{1-\alpha_{1} m^{2}}\right)\right] .
$$

Приращения деформаций $d \varepsilon_{a}=d a / a$ и $d \varepsilon_{h}=d h / h$ удовлетворяют уравнению неразрывности

соотношению

$$
d \varepsilon_{a}+d \varepsilon_{h}=\frac{d c}{1-c}
$$

$$
\frac{d m}{m}=d \varepsilon_{a}-d \varepsilon_{h},
$$

которое получается в результате дифференцирования величины $m=a / h$, и соотношениям (8) для макронапряжений:

$$
d \varepsilon_{a}=d \lambda \frac{\bar{\sigma}_{z}-\left(1-2 \alpha_{1}\right) \bar{\sigma}_{z}}{2\left(1-\alpha_{1}\right)}, d \varepsilon_{h}=d \lambda \frac{\bar{\sigma}_{z}-\left(1-2 \alpha_{1}\right) \bar{\sigma}_{z}}{2\left(1-\alpha_{1}\right)}
$$

Из (14) и (16) определяется коэффициент

$$
d \lambda=\frac{\left(1-\alpha_{1}\right) d c}{\alpha_{1}(1-c) \bar{\sigma}_{z}} .
$$

Подставляя (16) в (15), с учетом (13) и (17), получаем

$$
\frac{d m}{m}=\left(1-\frac{1}{\alpha_{1}}\right)\left(1-\frac{m}{\omega}\right) \frac{d c}{1-c},
$$




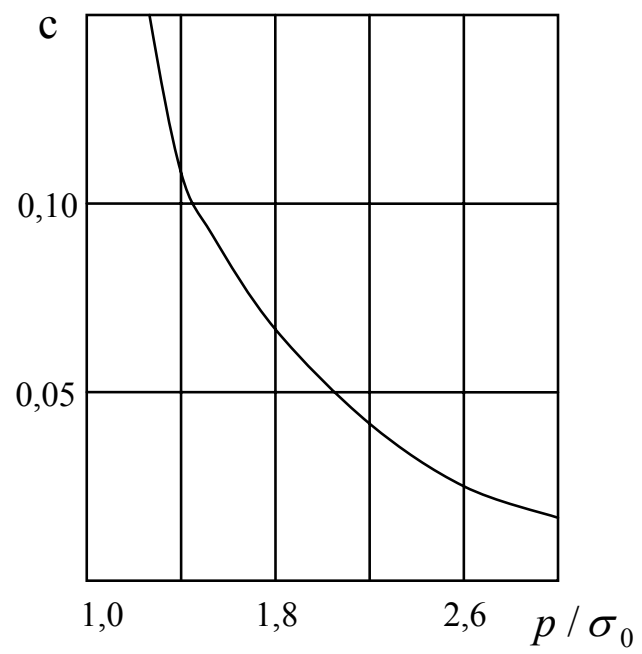

Р и с. 2. Изменение пористости шайбы при осадке в зависимости от давления где

$$
\omega=\left(1-\alpha_{1}\right) m+\sqrt{1-\alpha_{1}}\left(1+\sqrt{1-\alpha_{1} m^{2}}\right) .
$$

Решение (14) с начальными условиями $a=a_{0}$, $h=h_{0}$ при $c=c_{0}$ дает

$$
a h=\frac{a_{0} h_{0}(1-c)}{1-c_{0}}
$$

Соотношения (13), (18) и (20) составляют полную систему уравнений, определяющих изменение пористости и размеров шайбы в процессе сжатия в зависимости от среднего давления $p=-\bar{\sigma}_{z}$. На рис. 2 изображена кривая изменения пористости в зависимости от среднего давления. Значения пористости и давления рассчитаны по указанным формулам.

Таким образом, в результате рассмотрения процесса осадки круглой шайбы из пористого пластического

материала при сжатии между двумя матрицами получена замкнутая система уравнений, описывающих изменение пористости и размеров шайбы в зависимости от среднего давления.

\section{БИБЛИОГРАФИЧЕСКИЙ СПИСОК}

1. Гоффман О., Закс Г. Введение в теорию пластичности для инженеров. М.: Машгиз, 1957. 279 с.

2. Лаптев А. М. Закономерности осадки пористой полосы при наличии контактного трения // Обработка металлов давлением. Ростов-н-Д: РИСХМ, 1981. С. 76-81.

3. Смыслов А. Ю. К теории пластичности пористых сред // Изв. вузов. Машиностроение. 1980. .№ 4. C. $107-110$.

4. Смыслов А. Ю. К расчету напряжений осадки полосы из пористого материала // Изв. вузов. Машиностроение. 1990. № 2. С. 83-86. 